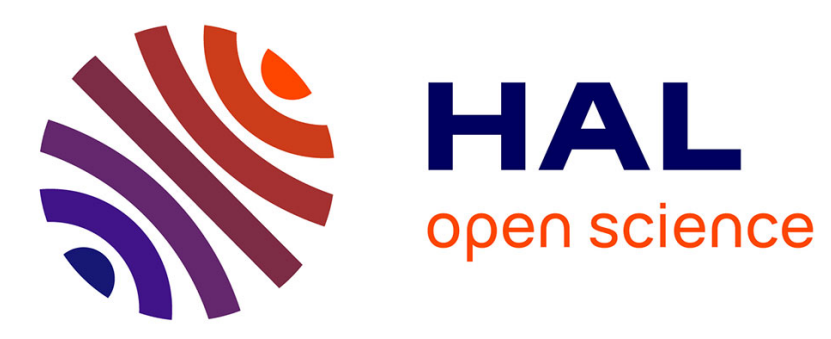

\title{
Pollution metallique relargable par les aerosols d'origine autoroutiere
}

Laurent Lebreton, Daniel R. Thevenot

\section{To cite this version:}

Laurent Lebreton, Daniel R. Thevenot. Pollution metallique relargable par les aerosols d'origine autoroutiere. Environmental Technology, 1992, 13, pp.35 - 44. 10.1080/09593339209385126 . hal01200588

\section{HAL Id: hal-01200588 \\ https://hal-enpc.archives-ouvertes.fr/hal-01200588}

Submitted on 16 Sep 2015

HAL is a multi-disciplinary open access archive for the deposit and dissemination of scientific research documents, whether they are published or not. The documents may come from teaching and research institutions in France or abroad, or from public or private research centers.
L'archive ouverte pluridisciplinaire HAL, est destinée au dépôt et à la diffusion de documents scientifiques de niveau recherche, publiés ou non, émanant des établissements d'enseignement et de recherche français ou étrangers, des laboratoires publics ou privés. 


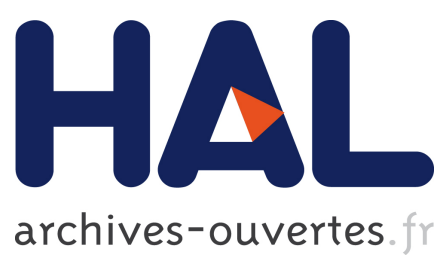

\section{POLLUTION METALLIQUE RELARGABLE PAR LES AEROSOLS D'ORIGINE AUTOROUTIERE}

Laurent Lebreton, Daniel R. Thévenot

\section{To cite this version:}

Laurent Lebreton, Daniel R. Thévenot. POLLUTION METALLIQUE RELARGABLE PAR LES AEROSOLS D'ORIGINE AUTOROUTIERE . Environmental Technology, Taylor \& Francis: STM, Behavioural Science and Public Health Titles, 1992, 13, pp.35-44. < hal-01179847>

\section{HAL Id: hal-01179847 \\ https://hal.archives-ouvertes.fr/hal-01179847}

Submitted on 23 Jul 2015

HAL is a multi-disciplinary open access archive for the deposit and dissemination of scientific research documents, whether they are published or not. The documents may come from teaching and research institutions in France or abroad, or from public or private research centers.
L'archive ouverte pluridisciplinaire $\mathbf{H A L}$, est destinée au dépôt et à la diffusion de documents scientifiques de niveau recherche, publiés ou non, émanant des établissements d'enseignement et de recherche français ou étrangers, des laboratoires publics ou privés. 


\title{
POLLUTION METALLIQUE RELARGABLE PAR LES AEROSOLS D'ORIGINE AUTOROUTIERE
}

\section{METAL POLLUTION RELEASE BY ROAD AEROSOLS}

\author{
LAURENT LeBRETON ET Daniel R. Thévenot*
}

Laboratcire de Bioélectrochimie et d'Analyse du Milieu (L.A.B.A.M.), Université Paris XII - Val de Marne, U.F.R. de Sciences et Technologie, Avenue du Général de Gaulle, 94010 Créteil Cedex, France.

(Received 18 March 1991; Accepted 7 November 1991)

\begin{abstract}
Because they are highly contaminated by heavy metals, road aerosols may pollute runoff waters. To estimate the mobility of some toxic metals such as $\mathrm{Zn}, \mathrm{Pb}$ or $\mathrm{Cd}$, these aerosols have been submited to a range of sequential chemical extraction (chemical speciation) and to laboratory release experiments. Both chemical speciation and reactor experiments show similar metal behaviour. $\mathrm{Zn}$ and $\mathrm{Cd}$ are extremely mobile (60\% released) while $\mathrm{Pb}$, highly bound to particles, needs a change of ionic strength, $\mathrm{pH}$ or the presence of chelating agents (addition of $\mathrm{NaCl}, \mathrm{pH} 4$ buffer or N.T.A. respectively) to dissolve significantly.
\end{abstract}

\section{INTRODUCTION}

Les aérosols urbains et en particulier routiers contiennent généralement d'importantes quantités de métaux lourds $(1,2)$ et peuvent représenter une source significative de pollution pour les milieux aquatiques récepteurs lorsqu'ils y sont entraînés par les eaux de précipitation et de ruissellement. Les formes physico-chimiques et les types d'associations que contractent ces polluants métalliques avec les particules solides (3) conditionnent leur toxicité, leur biodisponibilité et leur mobilité. Chester et al. (4) ont montré que la force des liaisons entre métaux $(\mathrm{Pb}, \mathrm{Cu}, \mathrm{Zn} . .$.$) et aérosols est très$ nettement plus faible dans le cas de particules d'origines anthropogéniques (poussières urbaines, particules de combustion...) que dans celui de poussières d'origine naturelle (érosion). Ainsi, $d u$ fait de l'importance des retombées atmosphériques sèches en ville (environ $1 \mathrm{~g} \cdot \mathrm{m}^{-2} \cdot \mathrm{j}^{-1}(2)$ ) et de leur forte teneur en certains éléments métalliques $(5,6)$ très labiles (4) et hautement toxiques pour la vie aquatique (7), ces aérosols urbains constituent une source de contamination et de pollution non négligeable pour les milieux "naturels" récepteurs.

Le protocole de spéciation chimique (extractions séquentielles) auquel nous avons soumis ces poussières nous a permis d'estimer la mobilité potentielle des métaux lors d'éventuelles modifications physico-chimiques du milieu aquatique. Parallèlement, afin de compléter ces informations "théoriques", nous avons placé ces particules en milieu aqueux et avons suivi les concentrations en métaux dissous afin de mettre en évidence les transferts métalliques entre phase solide (poussières) et solution (phase liquide simulant le milieu naturel). Ces deux approches apparaissent très complémentaires : les extractions séquentielles nous indiquent, avec un matériel restreint, la sensibilité de différents métaux à divers facteurs physico-chimiques et, les résultats des expériences en réacteur nous permettent d'estimer la qualité des prédictions du schéma de spéciation et d'acquérir des informations supplémentaires sur les vitesses et les états d'équilibre.

\section{MATERIEL ET METHODES}

Préparation des matériels

Toute pièce ou instrument directement ou indirectement en contact avec le milieu d'expérience subit un nettoyage poussé au détergent et à l'acide nitrique $30 \%$ suivi de rinçages avec de l'eau bidistillée purifiée par un appareil Millipore "Milli-Q". 


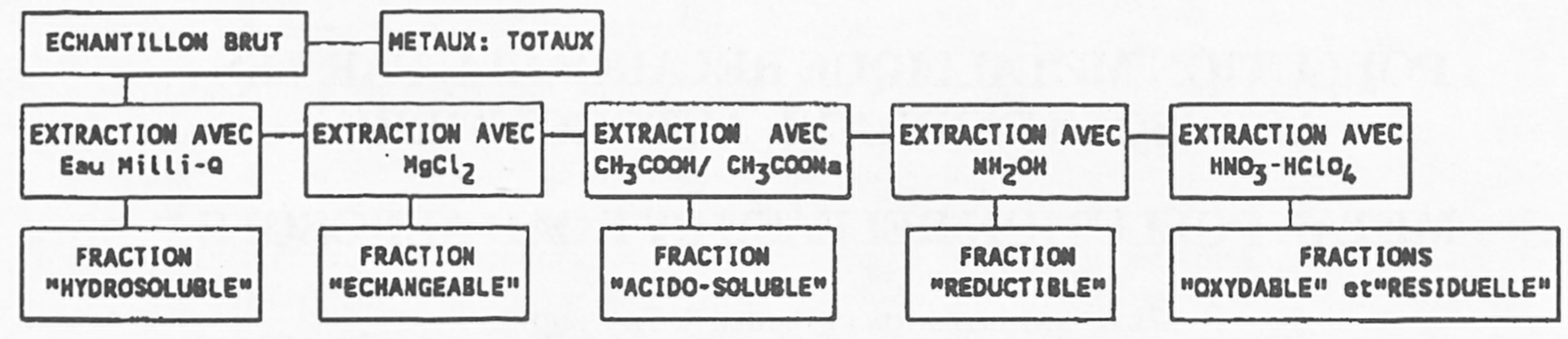

Figure 1. Schéma de spéciation chimique utilisé pour cette étude.

Prélèvement des aérosols

Une masse d'environ $200 \mathrm{~g}$ d' aérosols a été prélevée dans un tunnel du boulevard périphérique parisien, au voisinage de la porte d'Asnières : le site de prélèvement est une passerelle supportant des ventilateurs, située à environ $5 \mathrm{~m}$ de la chaussée et $2 \mathrm{~m}$ du plafond de ce tunnel. Ces poussières sont extrêmement fines, presque sèches $(8,5 \%$ d'humidité) et n'ont pas été en contact avec les eaux de pluie depuis leur dépôt sur la passerelle.

Caractérisation des aérosols

\section{Humidité et matières volatiles}

Ces mesures ont été réalisées (deux réplicats) grâce à un four à moufle (Thermolyne type 48000$): 5 \mathrm{~h}$ à $125^{\circ} \mathrm{C}$ pour l'humidité $(8,60 \pm$ $0.35 \%)$ et $3 \mathrm{~h}$ à $550^{\circ} \mathrm{C}$ poụr les matières volatiles $(20,60 \pm 0,45 \%)$.

\section{Extractions séquentielles}

Les extractions séquentielles sont réalisées sur un minimum de deux réplicats avec des produits de qualité "Merck Suprapur" et de l'eau bidistillée purifiée par une unité Millipore "Milli-Q". Le schéma de spéciation est résumé dans la Figure 1.

Les protocoles expérimentaux appliqués à environ $1 \mathrm{~g}$ d'aérosols secs comportent les 6 phases suivantes:

a. Métaux Totaux : extraction à l'eau régale par 2 h d'ébullition dans $4 \mathrm{ml} \mathrm{HCl} 35 \%+16 \mathrm{ml}$ $\mathrm{HNO}_{3} 52 \%$, centrifugation et dosage des métaux dans le surnageant;

b. Fraction "Hydrosoluble" : $1 \mathrm{~h}$ d'agitation à température ambiante dans $20 \mathrm{ml}$ d'eau "ultra-pure", centrifugation, dosage des métaux dans le surnageant, attaque suivante sur le culot de centrifugation ;

c. Fraction "Echangeable" : $2 \mathrm{~h}$ d'agitation à température ambiante dans $20 \mathrm{ml}$ de $\mathrm{MgCl}_{2} 1$
$\mathrm{M}$ à $\mathrm{pH} 7$, centrifugation, dosage des métaux dans le surnageant, attaque suivante sur le culot de centrifugation ;

d. Fraction "Acido-Soluble" : $2 \mathrm{~h}$ d'agitation à température ambiante dans $20 \mathrm{ml}$ de $\mathrm{CH}_{3} \mathrm{CO}_{2} \mathrm{H}-\mathrm{CH}_{3} \mathrm{CO}_{2} \mathrm{Na} 1 \mathrm{M} \mathrm{pH} \mathrm{5}$, centrifugation, dosage des métaux dans le surnageant, attaque suivante sur le culot de centrifugation ;

e. Fraction "Réductible" : $5 \mathrm{~h}$ d'agitation à $85^{\circ} \mathrm{C}$ dans $20 \mathrm{ml}$ de $\mathrm{NH}_{2} \mathrm{OH}, \mathrm{HCl} 0,04 \mathrm{M}$ dans $\mathrm{CH} 3 \mathrm{CO} 2 \mathrm{H} 25 \%$, centrifugation, dosage des métaux dans le surnageant, attaque suivante sur le culot de centrifugation.

f. Fraction "Oxydable" et "résiduelle": chauffage une nuit à $105^{\circ} \mathrm{C}$ dans $10 \mathrm{ml} \mathrm{HNO}{ }_{3}$ concentré + $1 \mathrm{ml} \mathrm{HClO}_{4}$ concentré, reprise dans $20 \mathrm{ml}$ $\mathrm{HNO}_{3} 1 \mathrm{M}$ après siccité, centrifugation, dosage des métaux dans le surnageant.

\section{Expériences en réacteur}

Nous avons utilisé un "réacteur" en verre rempli d'environ $500 \mathrm{ml}$ de solution, thermostaté à $20^{\circ} \mathrm{C}$ et agité en continu par un barreau magnétique et une recirculation de liquide assurée par une pompe péristaltiques Millipore "XX8000230" (débit environ $200 \mathrm{ml}^{\mathrm{mn}}{ }^{-1}$ ). Une sonde multifonction (HORIBA Water Checker U.7) a permis le suivi, à l'extérieur du réacteur (volume hors réacteur: environ $100 \mathrm{ml}$ ), des principaux paramètres physico-chimiques $(\mathrm{pH}$, $\mathrm{O}_{2}$ dissous, température, conductivité, M.E.S.). Les fractions à analyser ont été prélevées à la seringue dans le réacteur, filtrées sur une membrane en téflon de $0,45 \mu \mathrm{m}$ de porosité (Millipore HVLP) et conservées à $4^{\circ} \mathrm{C}$ avant analyse.

\section{Analyses effectuées}

Sur chacun des échantillons prélevés et filtrés, nous avons recherché et quantifié un certain nombre de polluants métalliques 
usuellement présents dans les eaux de ruissellement urbain $(\mathrm{Zn}, \mathrm{Pb}$ et $\mathrm{Cd}$, éventuellement $\mathrm{Cu}$ ), et mesuré les concentrations en $\mathrm{Ca}$ et $\mathrm{Mg}$ (cations majeurs influant sur la force ionique et compétiteurs des métaux lourds pour l'adsorption sur les sites de fixation) et, en $\mathrm{Fe}$ et $\mathrm{Mn}$ (indicateurs de la présence d'oxydes et hydroxydes de $\mathrm{Fe}$ ou $\mathrm{Mn}$, qui présentent de grandes surfaces d'adsorption). Ces analyses ont été effectuées à l'aide d'un spectrophotomètre d'absorption atomique en mode flamme ou four selon les concentrations recherchées (spectrophotomètre Perkin-Elmer modèle 1100 B associé au four HGA 700 et au passeur d'échantillon AS 70).

Les matières carbonées dissoutes ou colloïdales, de diamètre inférieur à $0,45 \mu \mathrm{m}$, étant susceptibles d'adsorber ou complexer les métaux lourds, nous avons suivi l'évolution de ce paramètre grâce à un analyseur de carbone organique et minéral (Astro modèle 2001).

Après stabilisation de la température du réacteur à $20^{\circ} \mathrm{C}$ et de la teneur en $\mathrm{O}_{2}$ dissous (saturation) dans la phase liquide (eau Milli-Q seule ou additionnée de divers composants ), nous introduisons une masse d'aérosol voisine de $5 \mathrm{~g}$ dans $600 \mathrm{ml}$ de solution. Quatre expériences ont été réalisées avec 4 solutions aqueuses de composition différente :

- Expérience 1 : Eau Milli-Q (pH voisin de 6).

- Expérience 2 : Eau Milli-Q avec 2 g.l $^{-1}$ de $\mathrm{NaCl}$ (Prolabo R.P. Normapur) ( $\mathrm{pH}$ voisin de 6 ).

- Expérience 3 : Eau Milli-Q ( $500 \mathrm{ml}$ ) + tampon $\mathrm{pH} 4(100 \mathrm{ml}$ de tampon potassium hydrogénophtalate 0,05 M Prolabo) ( $\mathrm{pH} 4$ ).

Expérience 4 : Eau Milli-Q avec 11,2 mg.l-1 d'acide nitrilotriacétique (N.T.A.: Prolabo) ( $\mathrm{pH}$ voisin de 6).

\section{RESULTATS ET DISCUSSION}

Schéma de spéciation utilisé dans cette étude

Un nombre très important de schémas de spéciation a été développé et les extractions séquentielles n'étant jamais tout à fait spécifiques et sélectives, il est parfois délicat de procéder à des intercomparaisons entre résultats obtenus avec des protocoles différents. Le schéma retenu pour cette étude, fortement inspiré de protocoles dûment testés et largement utilisés $(5,8,9,10)$ nous affranchit en partie de ce problème. Notre schéma de spéciation est dérivé de celui développé par Morrison et Revitt (2) pour les eaux pluviales. Seules, l'addition d'une fraction hydrosoluble (comme chez Gupta et Chen ou Welté par exemple in (11)) et la séparation des fractions "acido-soluble" et "réductible" (comme indiqué dans le schéma de Tessier in (11)) l'en différencie.

L'addition d'une fraction extractible à l'eau se justifie pleinement pour des particules sèches n'ayant pas été en contact avec ce liquide depuis leur dépôt et, la fraction "acido-soluble" nous permet de distinguer les effets dûs à une éventuelle acidification du milieu (pluies acides, baisse de $\mathrm{pH}$ en réseau...) de ceux générés par un passage en anoxie. L'éventail des perturbations physico-chimiques les plus courantes susceptibles de remobiliser les polluants métalliques est donc, avec ces 5 fractions, totalement pris en considération.

Le tableau 1 ci-après présente les résultats de la spéciation exprimés en $\mu \mathrm{g} / \mathrm{g}$ de matière sèche. Ces poussières sont extrêmement chargées en métaux lourds ( 2 à 10 fois plus que les valeurs citées dans la littérature $(1,5,7)$ ) ce qui s'explique par leurs origines autoroutières

Tableau 1. Résultats de la spéciation chimique des aérosols routiers.

\begin{tabular}{|l|cc|cc|cc|}
\hline \multirow{2}{*}{$\begin{array}{l}\text { Concentration } \\
\text { en } \mu \mathrm{g} / \mathrm{g} \text { de poids sec) }\end{array}$} & \multicolumn{2}{|c|}{ Zinc } & \multicolumn{2}{c|}{ Plomb } & \multicolumn{2}{c|}{ Cadmium } \\
& Essai 1 & Essai 2 & Essai 1 & Essai 2 & Essai 1 & Essai 2 \\
\hline 1.Fraction "hydrosoluble" & 1270 & 1200 & 140 & 130 & 24.7 & 24.9 \\
2.Fraction "échangeable" & 220 & 240 & 1220 & 1320 & 3.7 & 3.7 \\
3.Fraction "acido-soluble" & 50 & 60 & 340 & 360 & 0.7 & 0.7 \\
4.Fraction "réductible" & 580 & 590 & 2190 & 2260 & 2.6 & 2.6 \\
5.Fraction "oxydable" & 230 & 240 & 1170 & 1080 & 0.4 & 0.7 \\
Somme des 5 fractions & 2350 & 2320 & 5050 & 5130 & 32.1 & 32.6 \\
\hline Total (extr. à l'eau régale) & \multicolumn{2}{|c|}{$2330 \pm 100$} & \multicolumn{2}{|c|}{$4800 \pm 300$} & & $26.6 \pm 0.2$ \\
\hline
\end{tabular}

* Concentrations totales en $\mathrm{Fe}, \mathrm{Mn}$ et $\mathrm{Cu}$ respectivement égales à : 50.000, 400 et $435 \mu \mathrm{g} / \mathrm{g}$ d'aérosols secs. 
( $\mathrm{Pb}$ des carburants $(6,12), \mathrm{Zn}$ et $\mathrm{Cd}$ des huiles (7)...) et par leur non exposition aux eaux météoriques.

Expériences de relargages de métaux par les aérosols

Les expériences en réacteur nous permettent d'estimer, en "pilote", l'importance de la pollution métallique dissoute générée par la mise en contact de solides contaminés et de phases aqueuses de caractéristiques physicochimiques diverses simulant les milieux naturels ou les réseaux d'assainissement récepteurs. L'interprétation des résultats fournis par ces expérimentations et leur comparaison avec ceux obtenus grâce à la spéciation chimique nous permettent de mieux mettre en évidence les transferts potentiels de métaux lourds des particules vers la phase dissoute. Les deux approches étant différentes et complémentaires, la comparaison de leurs résultats rend possible une interprétation plus juste des valeurs observées, facilite une éventuelle extrapolation aux phénomènes réels (comportement dans les milieux récepteurs) et permet de définir les limites de chacune. De plus l'étude des cinétiques de "relargage" apparaît seule capable de fournir des éléments sur les vitesses des réactions et sur le temps nécessaire avant l'atteinte des équilibres entre pollution métallique "dissoute" et "particulaire", informations essentielles à l'estimation de l'impact environnemental d'un afflux de solides contaminés en milieu aquatique.
Fractions hydrosoluble et échangeable

La répartition des éléments métalliques entre les différentes fractions du schéma de spéciation (Fig. 2) met en évidence une nette prédominance des formes hydrosolubles, très labiles (les plus dangereuses pour l'environnement), pour $\mathrm{Zn}$ et $\mathrm{Cd}$. Ce résultat est comparable à ceux de Chester et al. (4) mais, dans le cas de poussières ayant été en contact avec les eaux météoriques ou de ruissellement $(5,7)$, il semble que ces fractions aient été en grande partie entraînées. $\mathrm{Pb}$ apparaît nettement moins labile et est quasiment insoluble dans l'eau pure. La faible mobilité de cet élément, fortement lié aux particules, a été observée par de nombreux auteurs (1,3,5,13-16). De telles poussières en contact avec des eaux météoriques "pures" tendraient donc, relativement, à s'appauvrir davantage en $\mathrm{Zn}$ et $\mathrm{Cd}$ qu'en $\mathrm{Pb}$. Ces résultats théoriques sont d'ailleurs confirmés par les expériences de relargage puisque, en présence d'eau Milli-Q seule, les proportions de $\mathrm{Pb}$ solubilisé restent toujours très inférieures ( 5 à 20 fois moins) à celles de $\mathrm{Zn}$ ou de $\mathrm{Cd}$ (Fig. 3).

En réacteur, la sensibilité du cadmium à l'augmentation de la force ionique par addition de $\mathrm{NaCl}$ est remarquable (Fig. 4), elle dépasse même nettement ce que prévoyaient les résultats de la spéciation : dans ce cas $100 \%$ du Cd est solubilisé en réacteur contre environ $89 \%$ prévus par la spéciation chimique pour la somme des fractions hydrosoluble et échangeable. Cet élément manifeste une préférence nettement

Répartition des métaux entre fractions \%
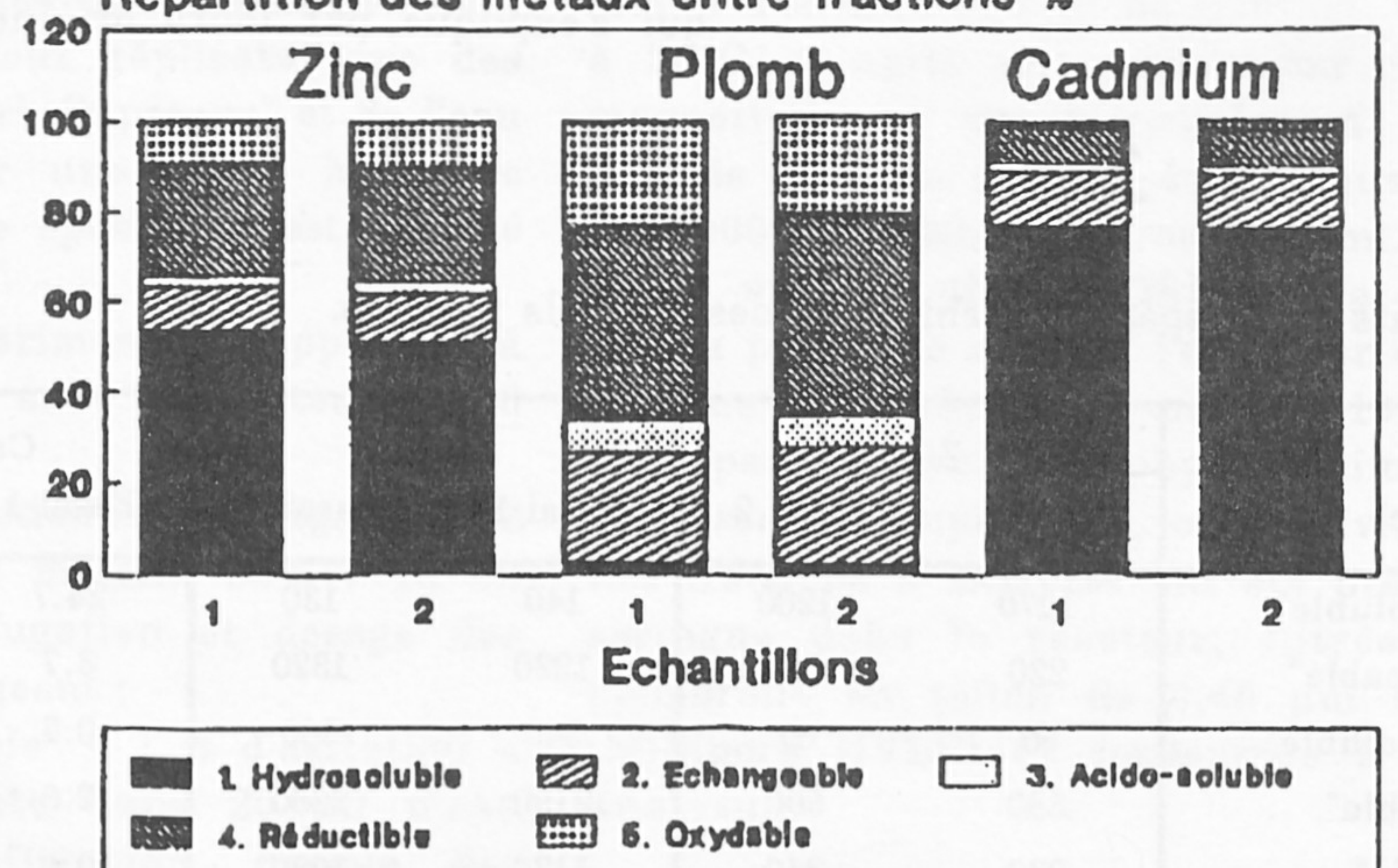

Figure 2. Importances relatives des différentes fractions de $\mathrm{Zn}, \mathrm{Pb}$ et $\mathrm{Cd}$ dans les aérosols soumis au schéma de spéciation décrit à la Figure 1. 

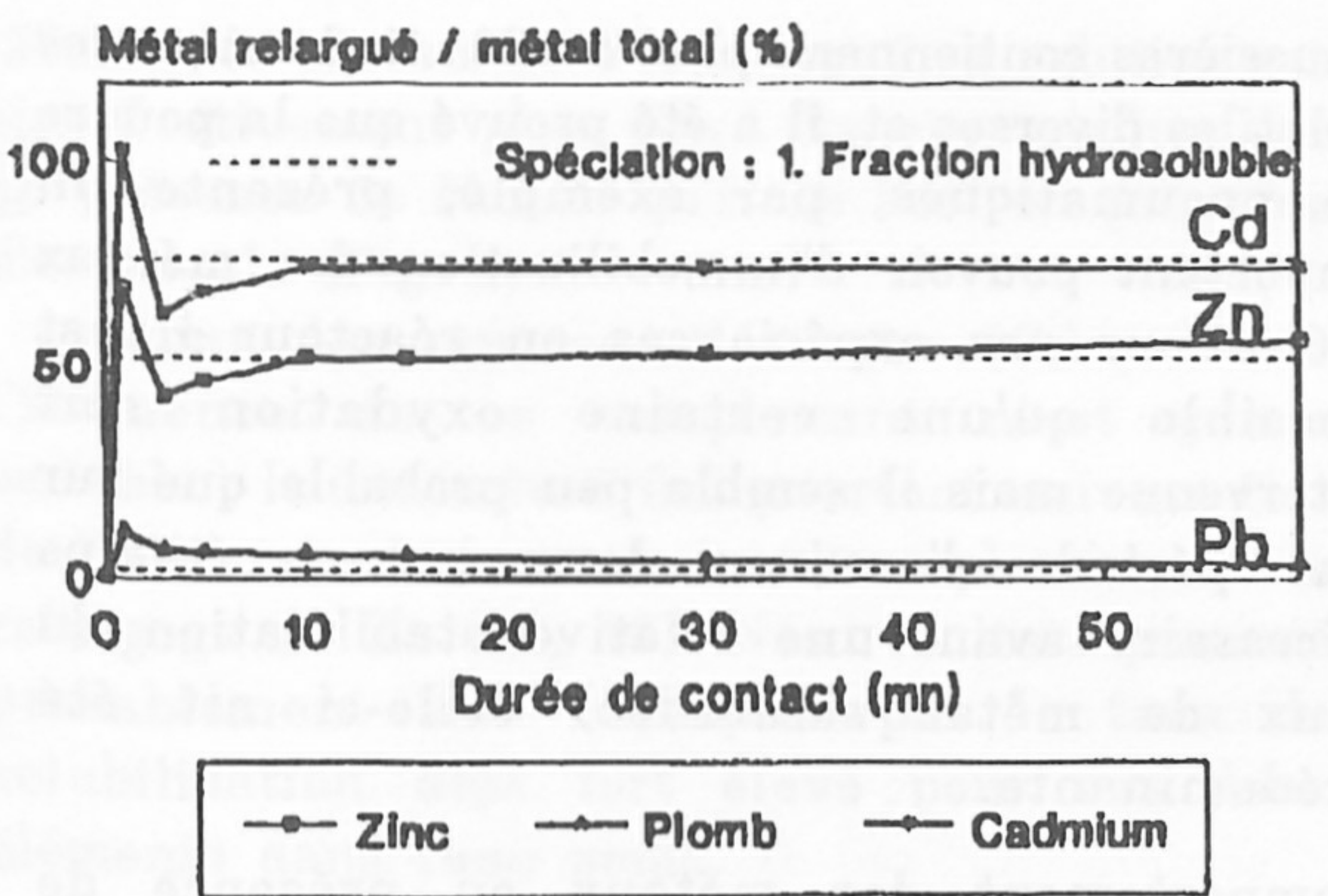
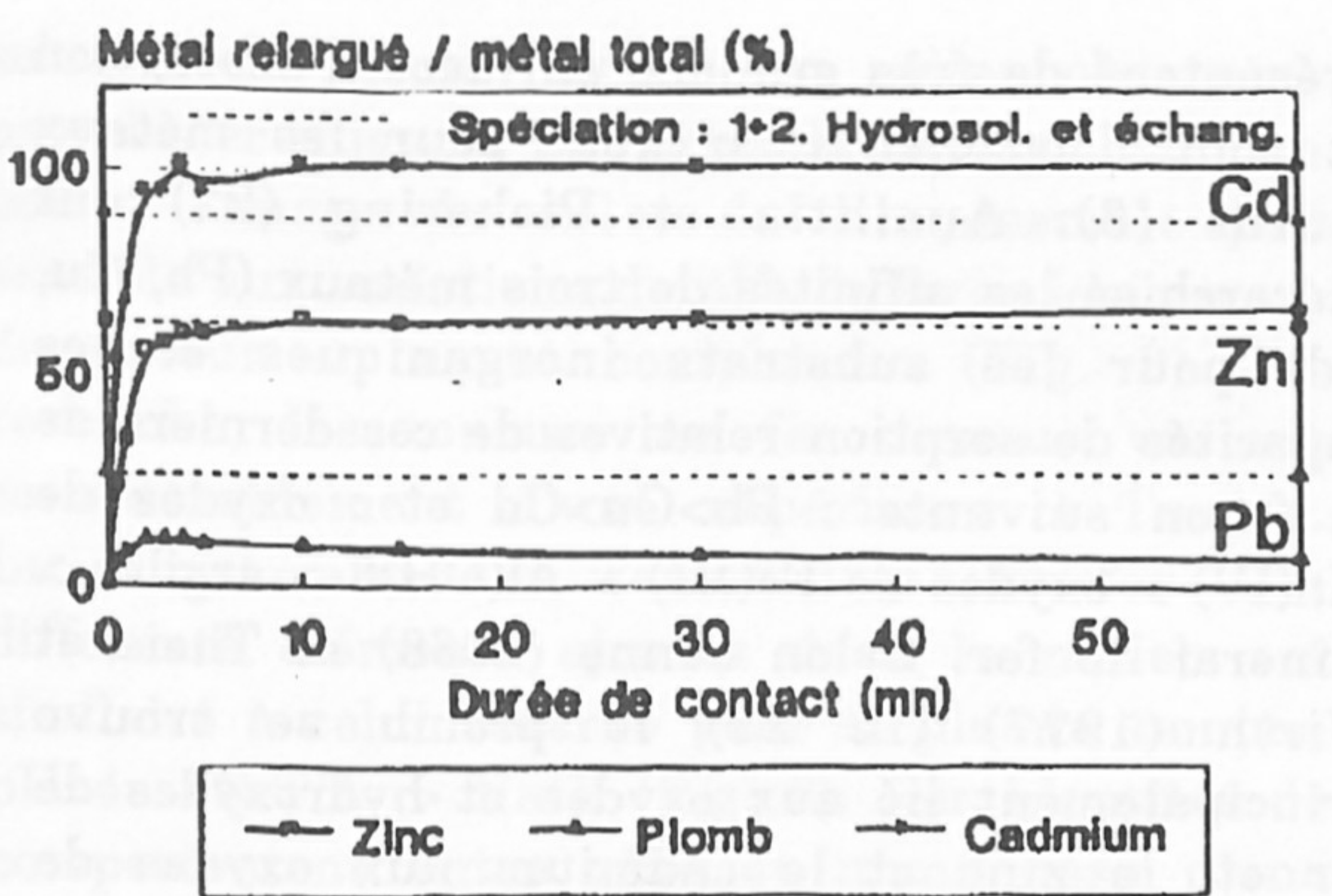

Figures 3 et 4 . Relargage des métaux contenus dans les aérosols lorsqu'ils sont placés à environ 4,5 g. $l^{-1}$ dans l'eau Milli-Q (Fig. 3) et dans l'eau additionnée de 2 g. $l^{-1}$ de $\mathrm{NaCl}$ (Fig. 4). Ces relargages sont exprimés par référence aux quantités totales de métaux déterminées par attaque à l'eau régale. Ils sont comparés aux résultats des étapes 1 ou 1 et 2 de la spéciation des aérosols (---) décrits par la Figure 1.

plus marquée pour les sites d'échange d'ions que pour tout autre type de site disponible sur ou dans les particules (17). Il n'est pas impossible que l'addition de chlorures entraîne une remobilisation du cadmium par complexation, les complexes chlorés du cadmium étant plus stables que ceux du zinc. A l'inverse, la spéciation sur-estime le pourcentage de $\mathrm{Pb}$ solubilisé (de 7 à $12 \%$ observé en réacteur contre $27 \%$ prévus pour les phases hydrosoluble et échangeable). En ce qui concerne $\mathrm{Zn}$, les deux résultats concordent presque parfaitement (65\% du $\mathrm{Zn}$ solubilisé). Tison (17) avait également observé la beaucoup plus grande sensibilité du cadmium que du plomb à l'augmentation de la force ionique (addition de $\mathrm{Na}^{+}$ou de $\mathrm{Ca}^{++}$). Les différences parfois constatées entre résultats de la spéciation et du relargage peuvent s'expliquer par l'utilisation de sels différents, et à des concentrations différentes, pour la spéciation $\left(\mathrm{MgCl}_{2} 1 \mathrm{M}\right)$ et pour les expériences en réacteur ( $\mathrm{NaCl} 34,2 \mathrm{mM})$.

Fraction acido-soluble

Les fractions acido-solubles des 3 métaux toxiques étudiés ne représentent que $2(\mathrm{Zn}$ et $\mathrm{Cd}$ ) à $7 \%(\mathrm{~Pb})$ de la quantité totale de métaux présente dans les aérosols. Harrison et al. (1) et Hamilton et al. (5) font état de valeurs supérieures qui sont peut-être à attribuer aux plus fortes teneurs en carbonates des poussières collectées en zone ouverte. Ces résultats se retrouvent en partie dans les expériences en réacteur où l'on constate que le plomb est le seul élément notablement affecté par la baisse de $\mathrm{pH}$ de 6 à 4. Hakansson et al. (14) ont observé également cette beaucoup plus grande sensibilité à l'acidification du milieu pour $\mathrm{Pb}$ que pour $\mathrm{Zn}$ ou $\mathrm{Cd}$. Cependant, la solubilisation en réacteur de près de $40 \%$ du plomb total excède de près de 6 fois les prévisions de la phase acido-soluble du schéma de spéciation tout en étant voisine de la somme des 3 premières fractions du schéma de spéciation (Fig. 5). Peut-être existe-til des phénomènes de complexation par l'hydrogénophtalate du tampon de $\mathrm{pH}$. Il faut également noter que même si le $\mathrm{pH} 4$ choisi pour l'expérience est proche de celui des précipitations en zone urbaine $(2,18)$, le pouvoir tampon de ces dernières n'a rien de comparable ni avec celui du milieu préparé au laboratoire ni avec celui généré par les poussières qu'elles rencontrent. Il faut enfin noter que si les quantités de $\mathrm{Zn}$ relarguées à $\mathrm{pH} 4$ sont très voisines de la somme des trois premières fractions du schéma de spéciation, il n'en est pas de même pour $\mathrm{Cd}$ : le relargage de $\mathrm{Cd}$ à $\mathrm{pH} 4$ est voisin de celui observé dans l'eau Milli-Q et significativement inférieur à celui observé en présence de $\mathrm{NaCl}$ pourtant de force ionique voisine.

\section{Fraction réductible}

Près de la moitié du plomb est extraite par le milieu réducteur (extraction avec $\mathrm{NH}_{2} \mathrm{OH}$ ), ce que constatent d'ailleurs nombre d'auteurs, tant dans les sédiments (Patchineelam 1975 in (19), $(20,21))$ que dans les poussières $(1,5)$. Ces substances réductibles sont essentiellement constituées d'oxydes de $\mathrm{Fe}, \mathrm{Mn}$ (et $\mathrm{Al}$ ) qui 
présentent de très grandes surfaces d'adsorption et sont d'excellents "pièges" pour les métaux lourds (8). Aualiitia et Pickering (22) ont hiérarchisé les affinités de trois métaux $(\mathrm{Pb}, \mathrm{Cu}$, Cd) pour les substrats inorganiques et les capacités de sorption relatives de ces derniers de la façon suivante : $\mathrm{Pb}>\mathrm{Cu}>\mathrm{Cd}$ et : oxydes de $\mathrm{Mn}(\mathrm{IV})>$ oxydes de $\mathrm{Fe}(\mathrm{III})>\mathrm{Al}(\mathrm{OH}) 3>$ argiles $>$ minerai de fer. Selon Jenne (1968) et Theis et Wirth (1977) (in 23) le plomb se trouve principalement lié aux oxydes et hydroxydes de fer et, le zinc et le cadmium aux oxydes de manganèse.

Lors de nos expériences en réacteur, nous n'avons pas travaillé en anoxie où nous aurions $\mathrm{pu}$ obtenir la réduction chimique ou microbienne (24) des oxydes métalliques. De ce fait, il est impossible de comparer directement les résultats obtenus par les deux approches (spéciations et expériences en réacteur). Cependant, nous avons suivi la solubilisation du Fer et du Manganèse et avons pu remarquer les comportements assez similaires de $\mathrm{Pb}$ et $\mathrm{Fe}$, d'une part, et de $\mathrm{Zn}$, Cd et $\mathrm{Mn}$, d'autre part, ce qui est en accord avec certaines des hypothèses précédemment énoncées (in 23).

Fraction oxydable

Des 3 éléments étudiés, le plomb est celui qui semble présenter la plus grande affinité pour la matière oxydable. Il semble probable que la teneur en acides humiques, excellents complexants et adsorbants des traces métalliques (25), soit assez faible et ne permette pas d'expliquer ces résultats (22\% du $\mathrm{Pb}$ extrait présent dans cette fraction). En revanche, les poussières contiennent plus de $20 \%$ de matières volatiles diverses et, il a été prouvé que la poudre de pneumatiques, par exemple, présente un important pouvoir d'immobilisation des métaux (26). Pour nos expériences en réacteur il est possible qu'une certaine oxydation soit intervenue mais il semble peu probable que sur une période d'environ deux heures (temps nécessaire avant une relative stabilisation du taux de métal solubilisé) celle-ci ait été prédominante.

Comportement des métaux en présence de complexant

L'acide nitrilotriacétique, associé aux zéolites et aux polycarboxylates, est de plus en plus utilisé comme substitut aux polyphosphates dans les détergents commerciaux et se retrouve à des teneurs non négligeable dans les milieux aquatiques. Les complexants synthétiques (N.T.A., E.D.T.A.) forment des liaisons plus stables avec les métaux lourds qu'avec $\mathrm{Ca}^{++}$ou $\mathrm{Mg}^{++}$et sont donc susceptibles de remobiliser ces micro-polluants minéraux lorsque ceux-ci ne sont pas trop fortement liés aux particules $(27,28,29)$. Le N.T.A., contrairement à l'acide éthylènediaminetétraacétique (E.D.T.A.), est relativement bien dégradé par voie biologique (29) en acide acétique et glycine (28) et ce dernier composé présente des capacités de complexation trop faibles pour provoquer une remobilisation significative de polluants métalliques (30). Dans nos expériences en réacteur, il apparait qu'à des concentrations de l'ordre de $10 \mathrm{mg} \cdot \mathrm{l}^{-1}$, c'est à dire à des teneurs 5 à 10 fois supérieures à celles observées dans les eaux usées (1,5 à $3 \mathrm{mg} . \mathrm{l}^{-1}$
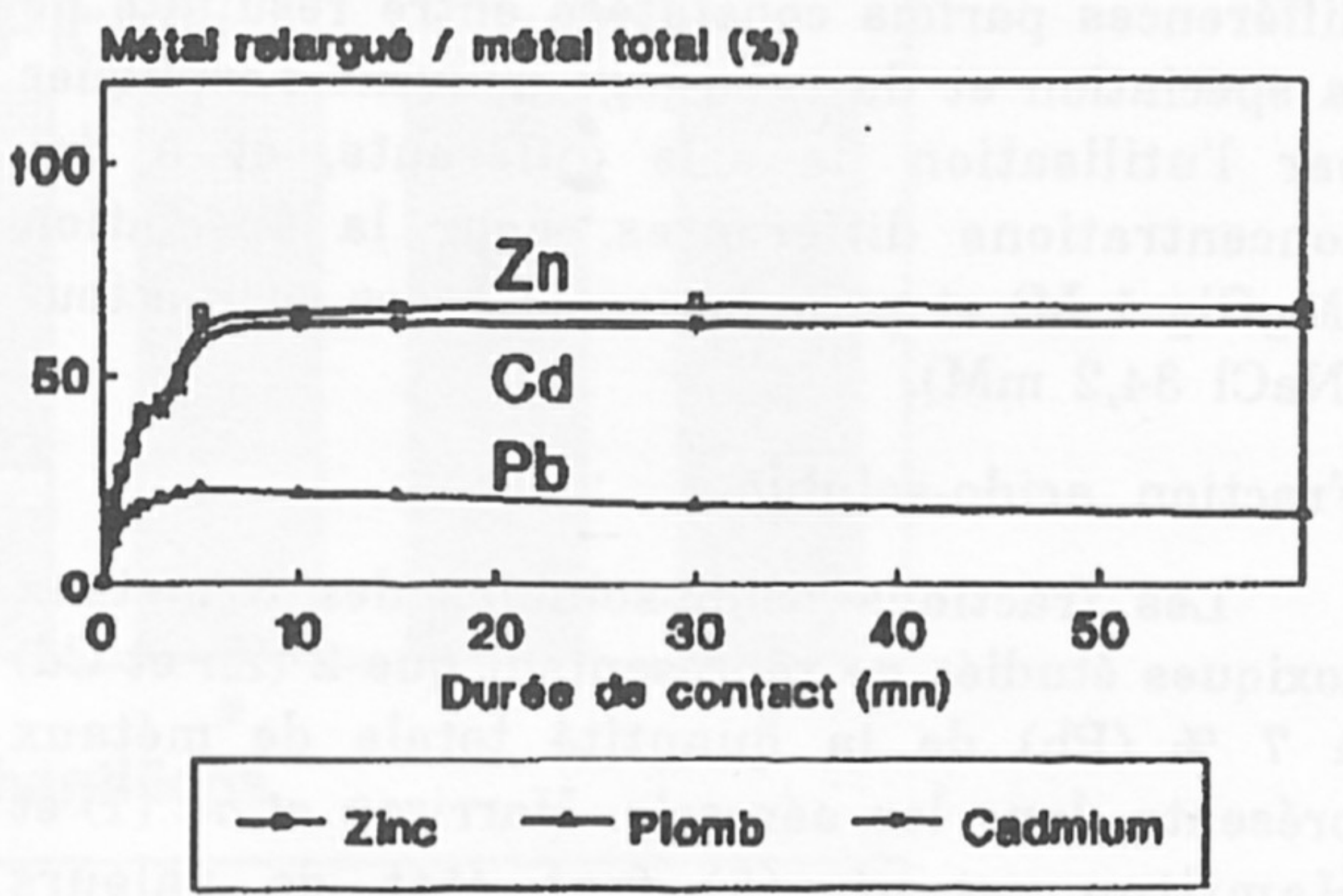

Figures 5 et 6 . Relargage des métaux contenus dans les aérosols lorsqu'ils sont placés à environ 4,5 g. l-1 $^{-1}$ dans l'eau tamponnée à $\mathrm{pH} 4$ (Fig. 5) et dans l'eau "Milli-Q" additionnée de N.T.A. (Fig. 6). Ces relargages sont exprimés par référence aux quantités totales de métaux déterminées par attaque à l'eau régale ; ils sont, dans la Figure 5, comparés à la somme des trois premières fractions du schéma de spéciation de la Figure 1 (---). 
$(27,29))$, le niveau du $\mathrm{Pb}$ relargué en solution est significativement plus élevé (teneur quadruplée) en présence de N.T.A. qu'il ne l'est en présence d'eau seule (Fig. 6).

Pour les autres éléments ( $\mathrm{Cd}$ et $\mathrm{Zn}$ ) l'influence de ce complexant n'est guère sensible; la littérature fait pourtant souvent état de l'effet très important de ce complexant sur le relargage de $\mathrm{Zn}(29,30)$. Nos résultats peuvent probablement être expliqués par le taux de solubilisation déjà fort élevé pour ces deux éléments dans l'eau seule.

Relargage du cuivre, du fer, du manganèse, du calcium, du magnésium et du carbone organique dissous

\section{Cuivre}

Cet élément présente un comportement assez différent selon les conditions physicochimique de milieu imposées (Fig. 7). Il semble particulièrement sensible à la présence de N.T.A. (environ $30 \%$ du $\mathrm{Cu}$ total solubilisé) et, dans une moindre mesure, à la baisse du $\mathrm{pH}$. Sa cinétique de relargage est relativement lente et l'équilibre "Aérosol-Solution" ne parait pas atteint après $90 \mathrm{mn}$ de brassage.

\section{Fer et Manganèse}

Nous n'avons pas recherché ces métaux lors de la spéciation chimique des aérosols, cependant, la littérature et d'autres de nos expériences présentent le manganèse comme un élément extrêmement mobile et le fer comme étant très peu solubilisable. Dans cette étude, malgré une teneur en manganèse total dans les aérosols environ 125 fois plus faible que celle en fer, cet élément présente des concentrations en solution du même niveau que celles observées pour le fer (Fig. 8). La solubilisation du Mn, un peu à l'image de celles du zinc, semble peu sensible aux variations de $\mathrm{pH}$, de force ionique ou de teneur en agent complexant (Fig. 8). En revanche, les teneurs en fer dissous varient considérablement d'une expérience à l'autre. Le classement des sensibilités du fer aux différents éléments ajoutés dans le milieu est similaire à celui observé pour le plomb: tampon pH $4>$ N.T.A. > NaCl (Fig. 8). Ces identités de comportement entre zinc et manganèse d'une part et, plomb et fer d'autre part peuvent être expliquées soit par des caractéristiques chimiques proches soit par une possible liaison de ces éléments deux à deux (oxydes et hydroxydes de fer-plomb; oxydes de manganèse-zinc).

\section{Cations majeurs $\left(\mathrm{Ca}^{2+}, \mathrm{Mg}^{2+}\right)$}

Il semble que les teneurs relevées dans les solutions mises en contact avec les aérosols routiers (teneurs en magnésium surtout) soient assez indifférentes aux modifications de milieu imposé $(\mathrm{NaCl}$, N.T.A., $\mathrm{pH})$ : ces teneurs en $\mathrm{Ca}$ et $\mathrm{Mg}$ sont respectivement comprises entre 300 et $350 \mathrm{~g} . \mathrm{l}^{-1}$ et 8 et $10 \mathrm{~g} . \mathrm{l}^{-1}$.

Carbone organique dissous

Si l'on assimilait les teneurs en C.O.T. dans les poussières à celles en Matières Volatiles (21\%), la solubilisation de C.O.T. correspondrait à $0,5 \%$ de sa teneur totale pour l'expérience $\mathrm{n}^{\circ} 2(\mathrm{Eau}+\mathrm{NaCl})$ et $3 \%$ pour l'expérience $\mathrm{n}^{\circ} 4$ (Eau + N.T.A.). Il faut toutefois noter que dans ce dernier cas la présence de N.T.A. (correspondant à $4,2 \mathrm{ppm}$ de carbone) provoque une importante augmentation de la
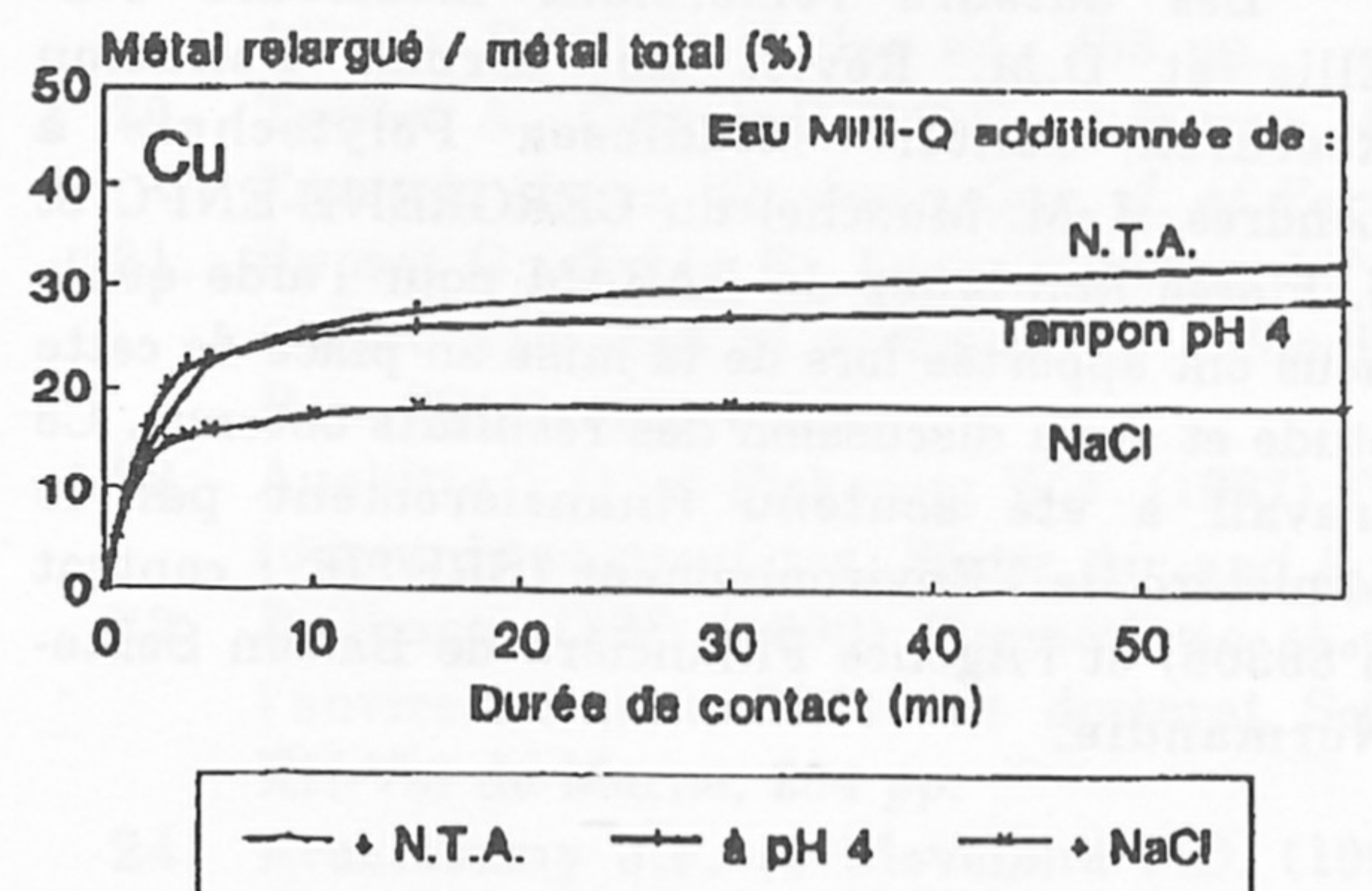
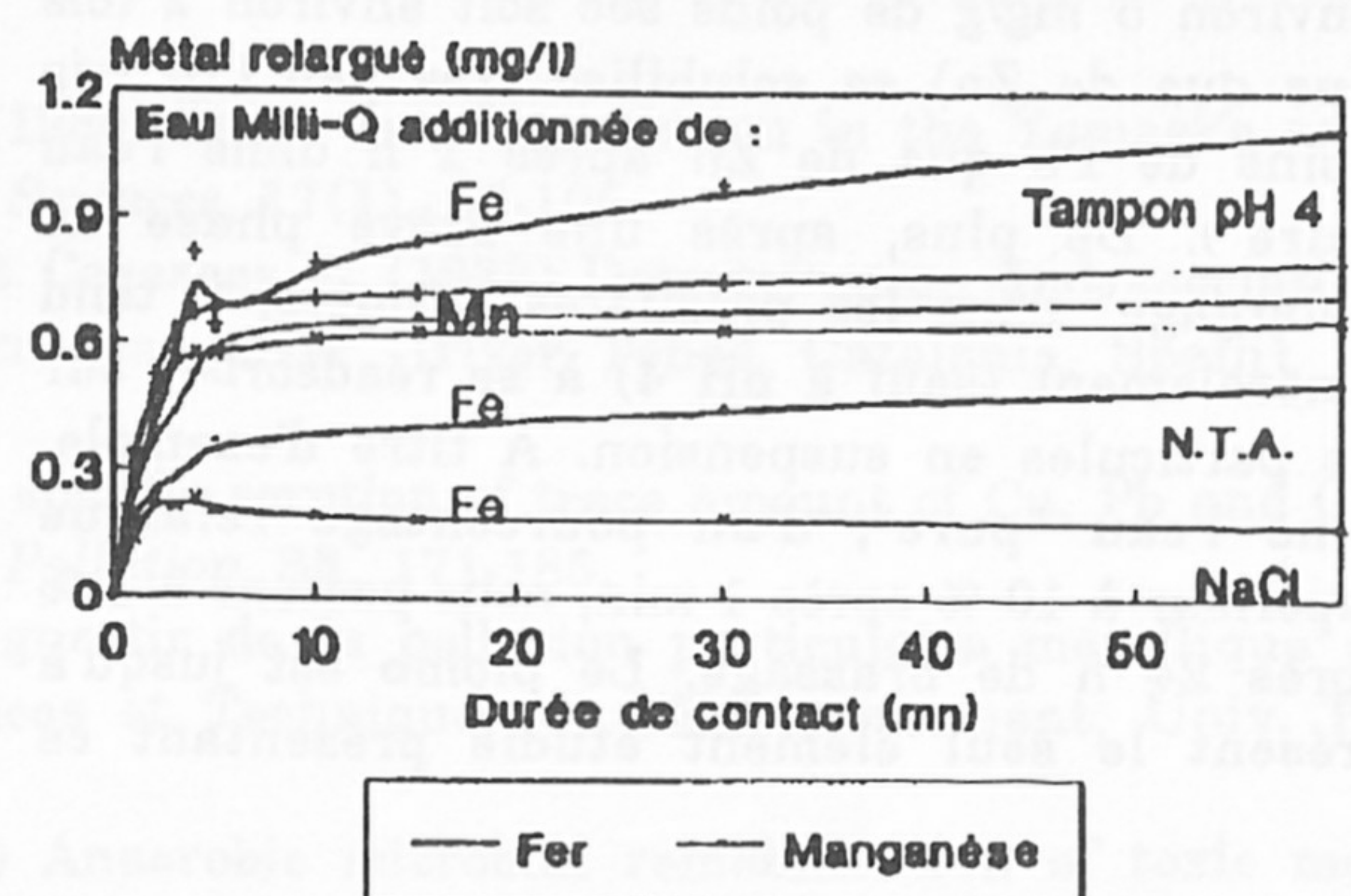

Figures 7 et 8 . Relargage de métaux contenus dans les aérosols lorsqu'ils sont placés à environ 4,5 g. $1^{-1}$ dans de l'eau Milli-Q additionnée soit de 2 g. $\mathrm{l}^{-1}$ de $\mathrm{NaCl}$, soit de tampon hydrogénophtalate $0,05 \mathrm{M}$ à $\mathrm{pH} 4$, soit de N.T.A. à 11 g.l ${ }^{-1}$ : relargage du Cu (Fig. 7) et de Fe \& Mn (Fig. 8) 
concentration en carbone organique dissous total (C.O.T. $=30 \mathrm{ppm}$ après $90 \mathrm{mn}$ ). Dans tous les cas, les teneurs en C.O.T. dissous augmentent avec le temps de contact des aérosols avec les solutions en suivant des cinétiques proches de celles observées pour les métaux (sauf pour le $\mathrm{Pb}$ ).

\section{CONCLUSION}

Zinc et Cadmium

Ces deux métaux s'avèrent extrêmement mobiles $(60 \%$ de la quantité totale de métaux initialement présents dans les aérosols routiers passe en solution en présence d'eau Milli-Q) et sont solubilisés en quelques minutes (équilibre atteint après $5 \mathrm{mn}$ ). En ce qui concerne $\mathrm{Zn}$, il apparaît que, si les conditions physico-chimiques sont modifiées (ajout de $\mathrm{NaCl}$, de N.T.A., de tampon $\mathrm{pH}$...), la proportion de métal relargué s'établit aux environs de $65 \%$ du métal total, soit environ $10 \%$ de plus que le relargage en présence d'eau "ultra-pure" seule. Cd est présent en quantité non négligeable ( $3,210-2$ mg.g-1) soit environ $1,4 \%$ de la teneur totale en $\mathrm{Zn}$. Ce chiffre est à comparer avec celui de la teneur naturelle en $\mathrm{Cd}$ du minerai de $\mathrm{Zn}$ qui est de l'ordre de $1 \%$ (12) : il n'existe donc probablement pas de pollution spécifique notable par cet élément dans ces aérosols routiers. Le comportement de Cd est assez proche de celui de $\mathrm{Zn}$ (très grande mobilité, vitesses de relargage et d'atteinte d'un équilibre "Eau-Sédiment" rapides), si ce n'est que ce métal semble particulièrement sensible à une variation de force ionique (eau $+\mathrm{NaCl}$ ).

\section{$\mathrm{Plomb}$}

Le plomb, bien qu'en quantité très importante dans les poussières routières (environ $5 \mathrm{mg} / \mathrm{g}$ de poids sec soit environ 2 fois plus que de $\mathrm{Zn}$ ) se solubilise très peu (14 fois moins de $\mathrm{Pb}$ que de $\mathrm{Zn}$ après $1 \mathrm{~h}$ dans l'eau "pure"). De plus, après une brève phase de "relargage" dans les premières minutes, il tend généralement (sauf à $\mathrm{pH}$ 4) à se réadsorber sur les particules en suspension. A titre d'exemple, dans l'eau "pure", d'un pourcentage relargué supérieur à $10 \%$ après $1 \mathrm{~min}$, nous passons à $1 \%$ après $24 \mathrm{~h}$ de brassage. Le plomb est jusqu'à présent le seul élément étudié présentant ce comportement. Les conditions d'agitation semblent avoir une grande influence sur sa cinétique de réadsorption ce qui peut expliquer en partie les moins bonnes corrélations entre résultats de la spéciation et des expériences en réacteur pour cet élément.

Le comportement de ce métal, tant lors de la spéciation chimique que lors des expériences en réacteur apparaît nettement différent de celui des deux éléments présentés précédemment. Les taux de solubilisation sont en effet très variables selon les types de modifications physicochimiques imposées (modification de la force ionique, du pH...). Ainsi, après une heure en réacteur, nous observons que $4 \%$ du plomb total est passé en solution si la phase liquide de départ est de l'eau Milli-Q seule, pourcentage qui passe à 7,16 et $38 \%$ si l'on ajoute respectivement $\mathrm{NaCl}$, N.T.A., ou du tampon pH 4 à cette phase liquide de référence.

Nous poursuivons et complétons ce travail par l'étude de l'influence des paramètres physiques et physico-chimiques sur le relargage des métaux associés aux sédiments transportés en réseaux d'eaux "pluviales". Ceci devrait nous permettre d'étayer ou de réviser nos hypothèses concernant les transferts de pollution métallique entre phases particulaires (poussières, sédiments...) souvent très contaminées (mais finalement relativement peu dangereuses pour les organismes vivants et les activités humaines) et phase liquide (eaux de surface ou souterraines) où la pollution métallique est directement accessible aux organismes aquatiques et aux pompages des usines de traitement d'eau.

\section{REMIERCIEMENTS}

Les auteurs remercient messieurs J.B. Ellis et D.M. Revitt du "Urban Pollution Research Center" Middlesex Polytechnic à Londres, J.-M. Mouchel du CERGRENE-ENPC et J. Flores Rodriguez du LABAM pour l'aide qu'ils nous ont apportée lors de la mise en place de cette étude et de la discussion des résultats obtenus. Ce travail a été soutenu financièrement par le Ministère de l'Environnement (SRETIE : contrat $n^{\circ} 89308$ ) et l'Agence Financière de Bassin SeineNormandie.

\section{BIBLIOGRAPHIE}

1. Harrison R.M., Laxen D.P.H. et Wilson S.J. (1981) Chemical associations of lead cadmium copper and zinc in street dusts and roadside soils, Environ. Sci. \& Technology, 15(11), 13781383. 
2. Morrison G.M.P. et Revitt D.M. (1987) Assessment of metal species bioavailibility and geochemical mobility in polluted waters, Sci. \& Techn. Lett., 8, 361-372.

3. Flores-Rodriguez J., Lebreton L., Bussy A.-L. et Thévenot D.R. (1990) Toxic metal speciation scheme for water and sediment from an urban storm sewage. 5th international conference on urban storm drainage, Osaka 1990, 457-462.

4. Chester, R. Lin F.J. et Murphy K.J.T. (1989) A three stage sequential leaching scheme for the characterisation of the sources and environmental mobility of trace metals in the marine aerosol. Environ. Techn. Lett., 10, 887-900 (1989).

5. Hamilton R.S., Revitt D.M. et Warren R.S. (1984) Levels and physico-chemical associations of $\mathrm{Cd} \mathrm{Cu} \mathrm{Pb}$ and $\mathrm{Zn}$ in road sediments, The Science of the Total Environment, 33, 59-74.

6. Bernard J.M. (1988) Routes et pollution de l'eau, Travaux 638, 40-44 (Décembre 1988).

7. Owe M., Craul J. et Halverson H.G. (1982) Contaminant levels in precipitation and urban surface runoff, Water Resources Bulletin, 18, (5), 863-868.

8. Tessier A., Campbell P.G.C. et Bisson M. (1979) Sequential extraction procedure for the speciation of particulate trace metals, Anal. Chem., 51(7), 844-851.

9. Ajayi S.O. et Vanloon G.W. (1989) Studies on redistribution during the analytical fractionation of metals in sediments, The Science of the Total Environment, 87/88, 171-187.

10. Belzile N., Lecomte P. et Tessier A. (1989) Testing readsorption of the trace element during partial extraction of bottom sediment. The Science of The Total Environment, 87/88, 171-187.

11. Flores-Rodriguez J. et Thévenot D.R. (1988) Etude de spéciation chimique des métaux lourds dans les eaux pluviales: Etude de faisabilité, RESEAU, Direction Régionale de l'Equipement, 75732 Paris, France, 86 pp.

12. Levesque J. (1978) Les micro-polluants minéraux dans les eaux superficielles continentales, rapports $n^{\circ} 3$ et 4: Le plomb, Le zinc-le cadmium, A.F.E.E.

13. Ellis J.B. et Revitt D.M. (1982) Incidence of heavy metals in street surface sediment: solubility and grain size studies, Water Air and Soil Pollution, 17, 87-100.

14. Hakansson K., Karlsson S. et Allard B. (1989) Effects of $\mathrm{pH}$ on the accumulation and redistribution of metals in a polluted stream bed sediment, The Science of the Total Environment, 87/88, 43-57.

15. Pereira-Ramos L. et Carru A.M. (1980) Comportement et bilan du cuivre du chrome et du plomb à la station d'épuration d'Achères, Trib. Eau, 41(534), 9-14.

16. Literathi P. et Laszlo F. (1976) Uptake and release of heavy metals in the bottom silt of recipients, Conference about sediment and freshwater exchanges, Holland, 403-409.

17. Tison A. (1985) Fixation des métaux lourds sur les sédiments: influence de la composition chimique du milieu aqueux, Thèse de doctorat Sciences et Techniques de l'environnement, Univ. Paris XII-Val de Marne, 127 pp.

18. Ng H.Y.F. (1987) Rainwater contribution to the dissolved chemistry of storm runoff, 4th international conference on urban storm drainage, Lausanne 1987, 21-26.

19. Förstner U. et Wittmann G.T.W. (1981) Metal pollution in the aquatic environment. Deuxième édition, Springer-Verlag éd., 486 pp.

20. Tessier A., Campbell P.G.C. et Bisson M. (1980) Trace metal speciation in the Yamaska and St François rivers (Quebec), Can. J. of Earth Sciences, 17(1), 90-105.

21. Rauret G., Rubio R., Lopez-Sanchez J.F. et Casassas E. (1988) Determination and speciation of copper and lead in sediment of a Mediterranean river (river Tenes, Catalonia, Spain), Wat. Res., $22(4), 449-455$.

22. Aualiitia T.U. et Pickering W.F. (1987) The specific sorption of trace amount of $\mathrm{Cu}, \mathrm{Pb}$ and $\mathrm{Cd}$ by inorganic particulates, Water Air and Soil Pollution, 35, 171-185.

23. Brilhante O.M. (1990) Magnétisme et diagnostic de la pollution particulaire métallique dans l'environnement, Thèse de doctorat Sciences et Techniques de l'Environnement, Univ. Paris XII-Val de Marne, 234 pp.

24. Aroklasamy J.F. et Cleveland J.D. (1990) Anaerobic microbial remobilisation of toxic metals coprecipited with iron oxide, Environ. Sci. Technol., 24(3), 373-378.

25. Seki H., Suzuki A. et Kashiki I. (1990) Adsorption of lead ions on immobilized humic acid, $J$. Colloid and Interface Sci., 134(1), 59-65 (1990).

26. Rowley A.G., Husband F.M. et Cunningham A.B. (1984) Mechanism of metal adsorption from aqueous solution by waste tyre rubber, Wat. Res., 18(8), 981-984. 
27. Lietz W. et Galling G. (1989) Metals from sediments, Wat. Res. 23(2), 247-252.

28. Mota A.M. et Gonçalvez M.L. (1990) NTA and lead speciation in natural water conditions, Wat. Res., 24(5), 587-590.

29. Alder A.C., Siegrist H., Gujer W. et Giger W. (1990) Behaviour of N.T.A. and E.D.T.A. in biological wastewater treatment, Wat. Res., 24(6), 733-742.

30. Huang C.P. et Hao O.J. (1989) Removal of some heavy metals by mordenite, Environ. Techn. Lett., 10, 863-874. 